\title{
CONTENEDORES, INSTRUMENTOS Y PIGMENTOS: UNA APROXIMACIÓN ARQUEOMÉTRICA A LOS PROCESOS DE PRODUCCIÓN Y USO EN LAS SOCIEDADES DEL HOLOCENO TARDÍO EN LOS HUMEDALES DEL SANTA LUCÍA, URUGUAY*
}

\author{
CONTAINERS, INSTRUMENTS AND PIGMENTS: AN ARCHAEOMETRIC \\ APPROACH TO THE PRODUCTION AND USE PROCESSES OF LATE \\ HOLOCENE SOCIETIES, SANTA LUCIA (URUGUAY) WETLANDS
}

\author{
Laura Beovide ${ }^{1}$, Maira Malán ${ }^{1}$, Elena Vallvé $^{1}$, Alejandro Trujillo ${ }^{2}$, Mirian Mejía $^{2}$, \\ Helena Pardo ${ }^{3,4}$, Ricardo Faccio ${ }^{3,4}$, Alvaro Mombrú ${ }^{3,4}$ y Mariela Pistón ${ }^{5}$
}

\begin{abstract}
Se expone una primera aproximación arqueométrica al estudio del uso de los pigmentos en el marco de la organización tecnológica de las sociedades prehistóricas del bajo río Santa Lucía. Se analizan, de forma exploratoria, la composición química y mineralógica de potenciales colorantes hallados en contextos arqueológicos y residuos de pigmentos en materiales cerámicos, aplicando las técnicas analíticas de Difracción de Rayos X (DRX), Espectroscopia Mössbauer por Transmisión (EMT) y Fluorescencia de Rayos $\mathrm{X}(\mathrm{FRX})$. Se ha podido identificar componentes mineralógicos como hematita $\left(\mathrm{Fe}_{2} \mathrm{O}_{3}\right)$ y goetita $(\mathrm{FeO}(\mathrm{OH}))$.

Palabras claves: pigmentos arqueológicos, espectroscopia, organización tecnológica, ocre.
\end{abstract}

This paper presents a first archaeometric approach to the study of the use of pigments in the context of the technological organization of prehistoric societies in Rio Santa Lucia. The chemical and mineralogical composition of potential colorants found in archaeological contexts and of pigment residues on ceramic and lithic materials are analyzed in an exploratory way, applying the analytical techniques of X-Ray Diffraction (XRD), Transmission Mössbauer Spectroscopy (TMS) and X-Ray Fluorescence (XRF). It was possible to identify mineral components such as hematite $\left(\mathrm{Fe}_{2} \mathrm{O}_{3}\right)$ and goethite $(\mathrm{FeO}(\mathrm{OH}))$.

Key words: Archaeological pigments, spectroscopy, technological organization, ochre.

En la cuenca inferior del río Santa Lucía, Uruguay (en adelante CISL, Figura 1), se han recuperado distintos pigmentos (Beovide 2011a) en contextos arqueológicos correspondientes a ocupaciones humanas de los últimos cinco milenios. Estos colorantes se presentan en forma de fragmentos (Figura 2), de artefactos de colorantes y como residuos en piedras con hoyuelos, manos y morteros (Beovide et al. 2007). Su uso se evidencia en la decoración de la cerámica (Malán et al. 2011), en los paquetes funerarios (Penino 1936) y en las pinturas rupestres ubicadas en la sierra hacia el norte de la cuenca (Peláez 1980).

Los pigmentos arqueológicos vienen siendo objeto de estudio en regiones vecinas con problemáticas comunes a la arqueología uruguaya a través de distintos abordajes arqueométricos (Di Prado et al. 2007; Matarrese et al. 2011; Porto y Mazzanti 2007; entre otros). Sin embargo, a pesar de haberse identificado pigmentos ("ocres") en diversos contextos prehistóricos del Uruguay su estudio espectrométrico ha quedado relegado.

* Artículo seleccionado del conjunto de ponencias presentadas en el III Congreso Latinoamericano de Arqueometría, realizado en Arica, Chile, el año 2011. Este manuscrito fue evaluado por investigadores externos y editado por Marcela Sepúlveda y Verónica Silva, en su calidad de editoras invitadas de la Revista.

1 Departamento de Arqueología, Museo Nacional de Antropología, Avda. de las Instrucciones 948, Montevideo, CP 12900, Uruguay.

2 Laboratorio de Arqueometría, Facultad de Ciencias Físicas, Universidad Nacional Mayor de San Marcos, Lima, Perú.

3 Centro Nano Materiales, Polo Tecnológico de Pando, Facultad de Química, Universidad de la República, Cno. Aparicio Saravia s/n 91000, Pando, Canelones, Uruguay.

4 Laboratorio de Cristalografía y Química del Estado Sólido (Cryssmat-Lab), DETEMA, Facultad de Química, Universidad de la República, Av. Gral. Flores 2124, Casilla de Correos 1157, Montevideo, Uruguay.

5 Laboratorio de Química Analítica, Departamento Estrella Campos, Facultad de Química, Universidad de la República, Gral. Flores 2124, P.O. Box 1157, Montevideo, Uruguay. 


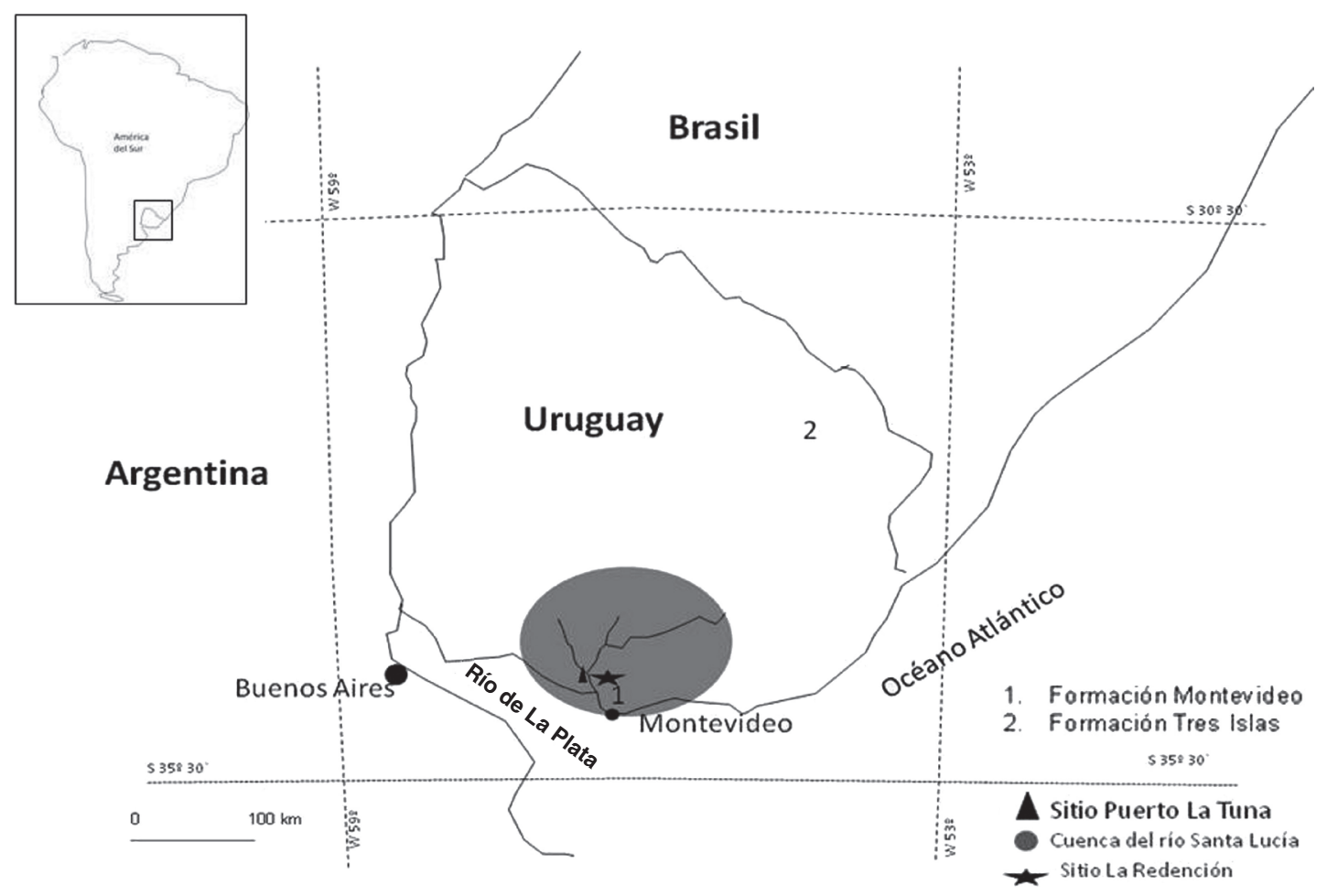

Figura 1. Mapa de ubicación: cuenca inferior del río Santa Lucía, sitios Puerto La Tuna y Redención. Location map: Inferior basin of the Santa Lucía River, and the Puerto La Tuna and Redención sites.

Como un primer avance al respecto, se propone explorar mediante distintas técnicas arqueométricas (DRX, FRX y Mössbauer) la composición química y mineralógica de colorantes recuperados para el Holoceno Tardío en la CISL y particularmente en el sitio Puerto La Tuna.

A nivel arqueológico el Sitio Puerto La Tuna ha sido caracterizado como sitio de actividades múltiples, entre las que destacan funebria y habitación. Respecto de la excavación VII, ubicada hacia el noreste del sitio, desde el punto de vista estratigráfico se reconoce un único componente cultural, siendo una ocupación asociada cronológicamente al momento de contacto hispano-indígena: (AMS) de 1.540 \pm 65 cal d.C. (Beovide 2011b; Malán et al. 2011). El material arqueológico recuperado comprende, entre otros, lítico tallado y pulido y cerámica, registrándose la totalidad de la cadena operativa tanto del sistema de producción lítico como cerámico (Lemos y Duarte 2011; Malán et al. 2011). Para el caso de la cerámica se recuperaron 943 fragmentos (el $85 \%$ no supera los $2 \mathrm{~cm}$ de lado) con diferentes tratamientos de superficie, destacándose la pintura monocroma roja en cara interna, externa o en ambas $(n=101)$. Formando parte de la cadena operativa también se recuperaron 18 pequeñas masas de arcilla, 124 pigmentos minerales (de 1 y $2 \mathrm{~cm}^{2}$, con una masa de 0,12 a $0,62 \mathrm{~g}$, con matices desde los amarillos $-5 \mathrm{Y}$ 8/8, escala Munsell- a los rojos -10 R 4/8, escala Munsell-, así como una mano y mortero fracturados con evidencias macroscópicas de pigmentos en su superficie. El hecho de que los pigmentos se pueden reconocer tanto en las piezas ya acabadas de la producción cerámica (vasijas pintadas), como en forma de desechos de esa producción (fragmentos de pigmentos, instrumentos pulidos utilizados en la elaboración de los colorantes) otorga singularidad a este sitio respecto de los otros estudiados en el área.

Con el objetivo de aportar al conocimiento de la organización tecnológica y a la discusión sobre aspectos territoriales vinculados a estrategias de aprovisionamiento, se llevan a cabo estudios comparativos de la composición mineralógica de fragmentos de colorantes y de cerámica pintada provenientes de la excavación VII del sitio Puerto 


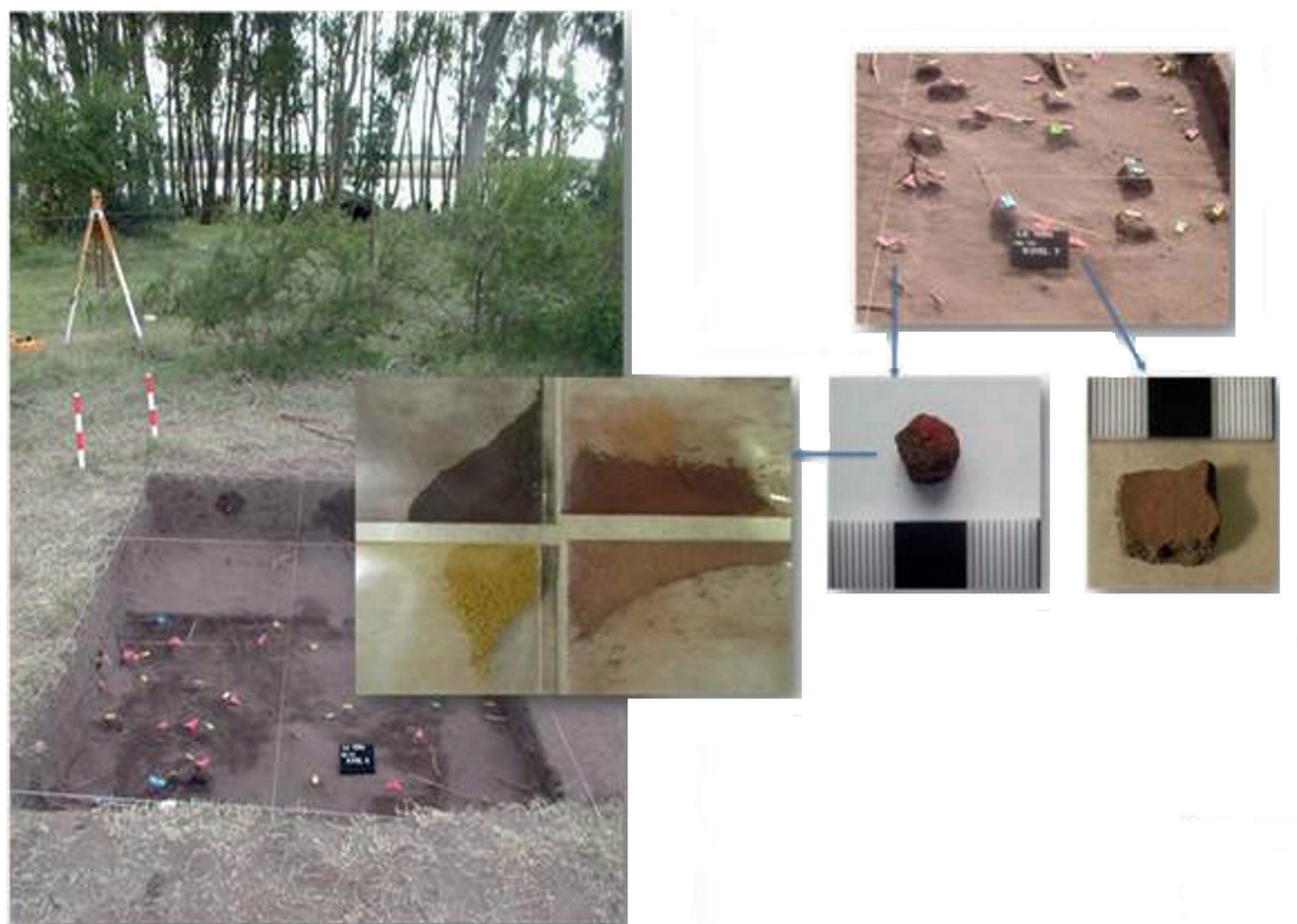

Figura 2. Imágenes de excavación VII (Puerto La Tuna). En detalle el contexto de identificación de pigmentos y cerámica. Puerto La Tuna, excavation VII images. Context details, with identification of pottery and pigments.

La Tuna, y de pigmentos de la misma excavación en relación con potenciales fuentes de abastecimiento.

\section{Materiales y Métodos}

\section{Muestras arqueológicas de pigmentos minerales}

Las muestras arqueológicas provienen de la excavación VII del sitio Puerto La Tuna y están asociadas a un fechado radiocarbónico (AMS) de $1.540 \pm 65$ cal d.C.

La muestra 2011-1(M3) corresponde a un fragmento de pigmento mineral de color amarillo $-5 \mathrm{Y}$ $8 / 8$, escala Munsell- mientras que las 2009-2(1) y 2011-5(2) corresponden a pigmentos minerales de color rojo -10 R 4/8, escala Munsell- (Tabla 1).

Las muestras 2009-1(M6) y 2011-4 (la tuna) corresponden a superficies pintadas de tiestos de cerámica de color rojo - $10 \mathrm{R}$ 4/8- y están asociadas espacialmente a los pigmentos minerales (Figura 2, Tabla 1).

\section{Muestras de potenciales fuentes de abastecimiento}

Las muestras de pigmentos potenciales provienen de los afloramientos de la Formación Montevideo en Punta Yeguas y de la Formación Tres Islas en Cerro Largo, ubicados respectivamente a $20 \mathrm{~km}$ y a $200 \mathrm{~km}$ del sitio arqueológico Puerto La Tuna. Ambas muestras, 2011-2 (Punta Yeguas) y 2011-3 (Tres Islas), corresponden a pigmentos minerales de color amarillo $-5 \mathrm{Y} 8 / 8$, escala Munsell- (Tabla 1).

\section{Instrumental}

Los perfiles de difracción de rayos $\mathrm{X}$ fueron obtenidos mediante el empleo de un equipo Rigaku, modelo Ultima IV, con monocromador CBO, operado a $40 \mathrm{kV}$ y $20 \mathrm{~mA}$, con un paso de $0,02 \% \mathrm{seg}$ $\mathrm{y}$ con la longitud de onda $\mathrm{CuK}_{\alpha}\left(\lambda_{1}=1.54056 \mathrm{y}\right.$ $\lambda_{2}=1.54439 \AA$ ), en el rango de $2 \theta=5.00$ a 50.00$70.00^{\circ}$ (según la muestra). 


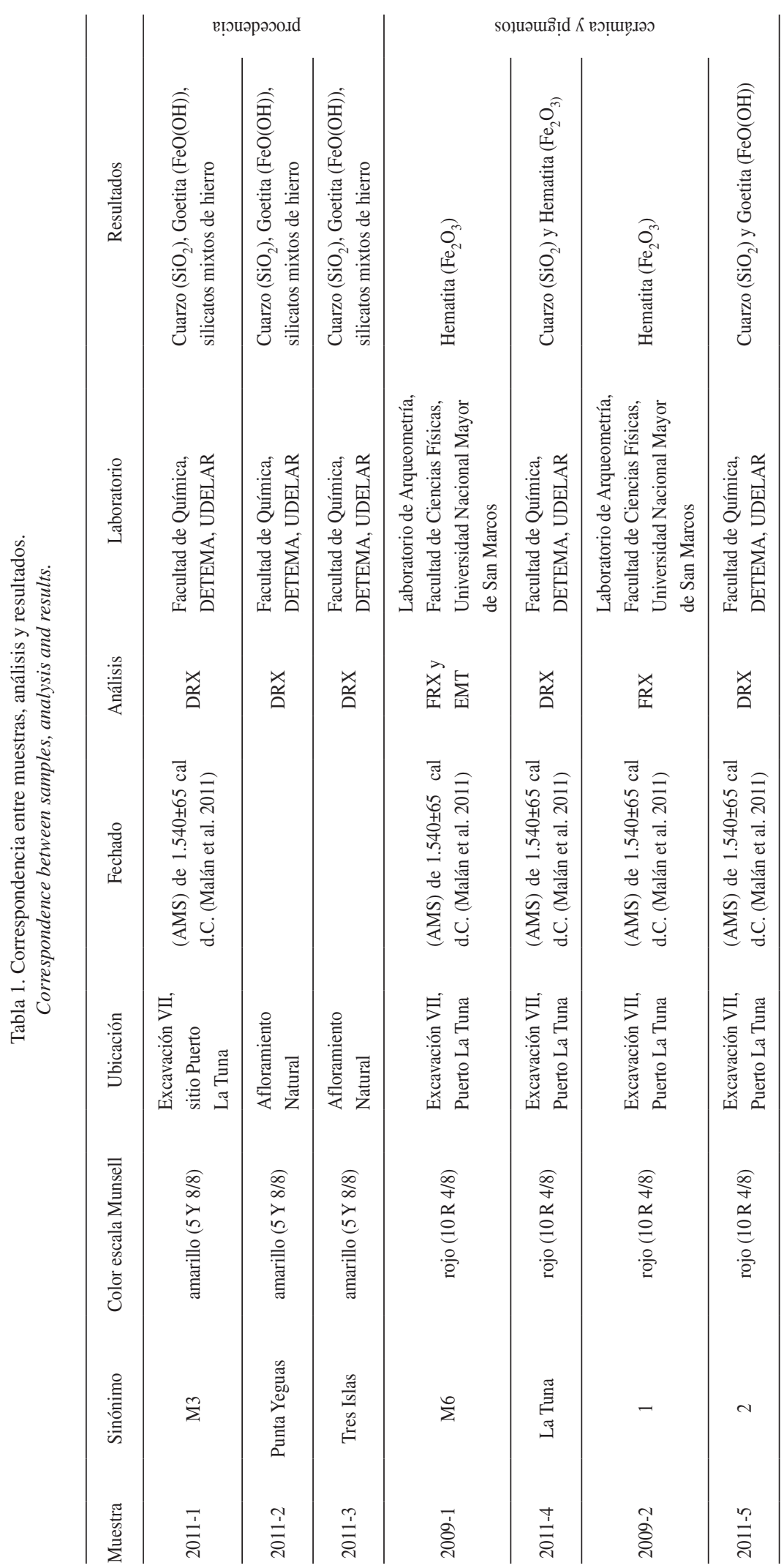


Las medidas de FRX se realizaron con un equipo portátil que utiliza un tubo de rayos $\mathrm{X}$ con un blanco de plata, operado a $25 \mathrm{keV}$, y un detector de estado sólido. También se utilizó un colimador de $2 \mathrm{~mm}$ de diámetro interno para el haz de rayos $\mathrm{X}$ incidente. Se trabajó con un ángulo de $45^{\circ}$ tanto para el haz incidente como reflejado.

El equipo de EMT empleado corresponde a un espectrómetro convencional, que utiliza una fuente radiactiva de Cobalto-57 en una matriz de rodio. La actividad promedio de la fuente es de $5 \mathrm{mCi}$. El equipo opera en un arreglo por transmisión y a aceleración constante.

\section{Método}

Para el estudio de potenciales fuentes de colorantes amarillos se compara el difractograma de rayos $\mathrm{X}$ de la muestra de pigmento arqueológico (2011-1) con los difractogramas de fuentes potenciales de minerales de la Formación Montevideo (muestra 2011-2) y de Formación Tres Islas (muestra
2011-3). Adicionalmente se realiza la comparación de los distintos diagramas obtenidos experimentalmente con diagramas compilados en bases de datos adecuadas.

Con el fin de buscar correlaciones entre los pigmentos arqueológicos y los tiestos de cerámica pintada del sitio Puerto La Tuna se analizan las muestras por DRX, FRX y EMT. Con DRX se analiza la superficie pintada de la muestra del tiesto de cerámica 2011-4 y el pigmento 2011-5. Con FRX se analiza la superficie pintada de la muestra del tiesto de cerámica 2009-1 y el pigmento 2009-2. A la muestra de cerámica 2009-1 se le realiza además EMT.

\section{Resultados}

\section{Fuentes potenciales de abastecimiento de colorantes y pigmentos arqueológicos}

A partir del análisis por DRX se puede concluir que las muestras 2011-1 al 3 (Figuras 3 y 4) tienen una composición mineralógica análoga

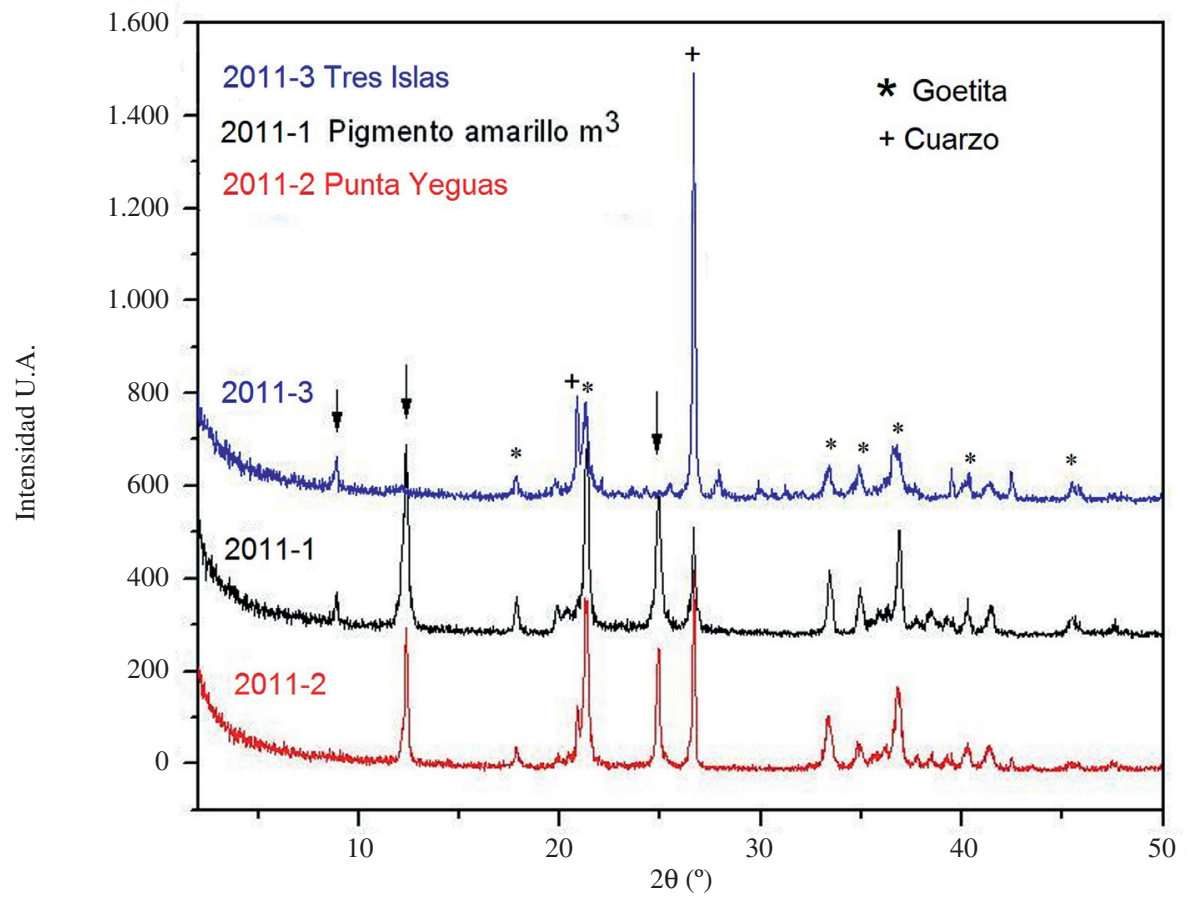

Figura 3. Comparación de los distintos diagramas de difracción obtenidos experimentalmente para las muestras naturales y la de pigmento arqueológico. De arriba hacia abajo: muestras de pigmento amarillo (2011-3, Tres Islas), (2011-1, $\left.\mathrm{m}^{3}\right)$ y (2011-2, Punta Yeguas). Las flechas indican los picos más importantes no presentes en todos los diagramas.

Comparison of different diffraction patterns obtained for the standards and the archaeological pigment samples. From top to bottom for yellow pigment samples: (2011-3, Tres Islas), (2011-1, $\left.\mathrm{m}^{3}\right)$ and (2011-2, Punta Yeguas). Arrows indicate most important diffraction peaks that are not present in all diagrams. 


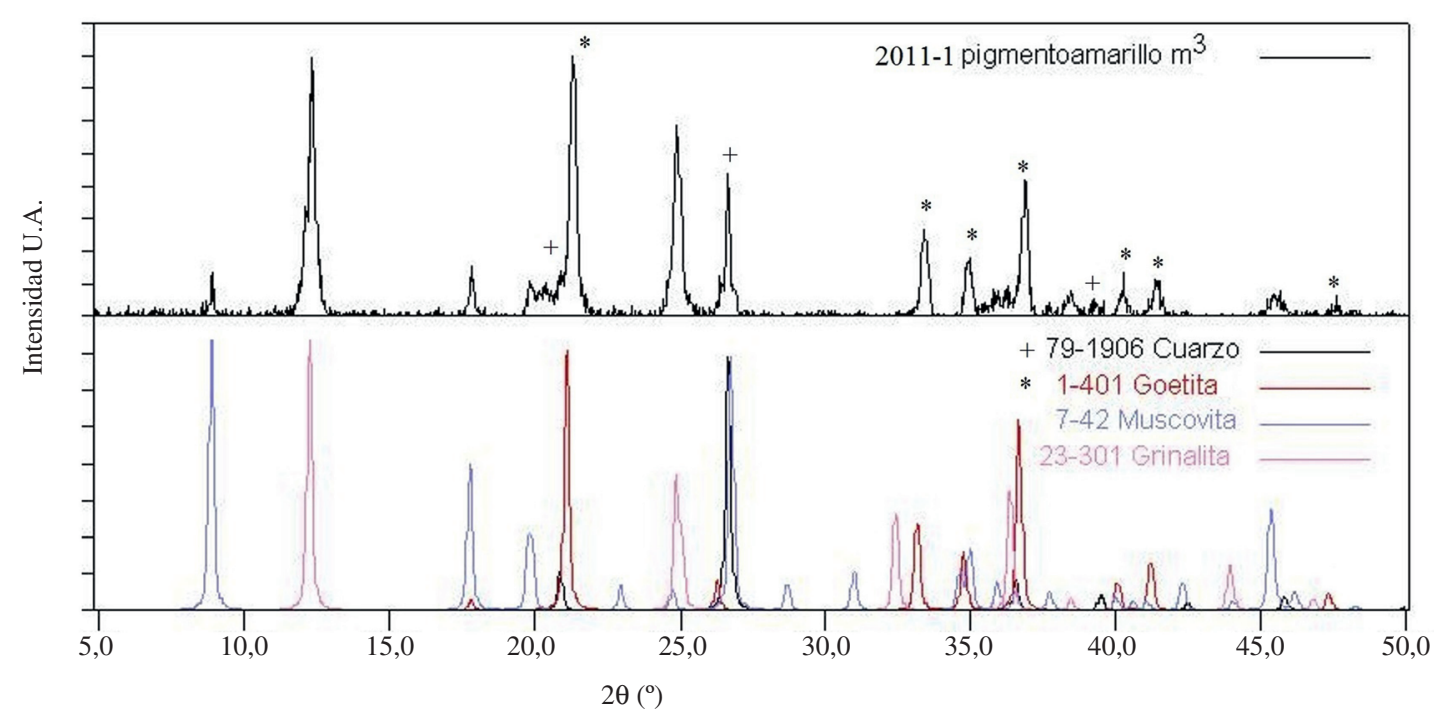

Figura 4. Diagrama de difracción experimental de $\mathrm{m}^{3}$ (superior) comparado con los de las muestras minerales de mayor coincidencia compilados en bases de datos (inferiores).

Experimental diffraction pattern of $\mathrm{m}^{3}$ (superior) compared with those of the best matching mineral standards compiled into databases (inferior).

compuesta mayoritariamente por cuarzo $\left(\mathrm{SiO}_{2}\right)$, goetita $(\mathrm{FeO}(\mathrm{OH}))$ y uno o más silicatos mixtos de hierro (muy posiblemente muskovita $(\mathrm{K}, \mathrm{Na})(\mathrm{Al}, \mathrm{Mg}, \mathrm{Fe})_{2}$ $\left(\mathrm{Si}_{3} \mathrm{Al}_{1}\right) \mathrm{O}_{10}(\mathrm{OH})_{2}$ y grinalita $\left.\left(\mathrm{Fe}_{3} \mathrm{Si}_{2} \mathrm{O}_{5}(\mathrm{OH})_{4}\right)\right)^{1}$. De estas muestras, las que exhiben mayor coincidencia entre sí son 2011-1 y 2011-2, correspondientes a un pigmento de origen arqueológico (excavación VII sitio Puerto La Tuna) y a otro pigmento proveniente de la Formación Montevideo en Punta Yeguas, potencial fuente de abastecimiento (Figura 3). Si bien exhiben ciertas diferencias, que pueden ser atribuidas al fenómeno de orientación preferencial, muestran un alto grado de coincidencia, lo que podría atribuirse a que fueron extraídas del mismo lugar.

\section{Fuentes potenciales de abastecimiento de colorantes y cerámica pintada}

A nivel macroscópico se puede apreciar que tanto el color rojizo como la textura terrosa observados en la muestra de cerámica arqueológica 2009-1 y en el pigmento 2009-2 (fuente potencial de abastecimiento) corresponderían a hematita $\left(\mathrm{Fe}_{2} \mathrm{O}_{3}\right)$. No se observa azufre (A) ni mercurio $(\mathrm{Hg})$ en los espectros por FRX (Figuras 5a y b) por lo que se descarta la presencia de cinabrio, lo que corroboraría que tanto en la superficie pintada de la cerámica como en el pigmento hay presencia de hematita. La Figura 5c corresponde al espectro
Mössbauer a temperatura ambiente de la muestra 2009-1; en él se puede apreciar una distribución de sitios magnéticos constituida por un conjunto de sitios cristalinos adjudicados a óxidos de hierro, probablemente distintos tipos de hematita. Asimismo se identifican dos sitios de hierro $\mathrm{Fe}_{3}+\mathrm{y}$ dos sitios de hierro $\mathrm{Fe}_{2}+$. Uno de los sitios de hierro $\mathrm{Fe}_{3}+$ es asignado a algún óxido de hierro en estado superparamagnético. Los otros sitios de hierro forman parte de la estructura de arcillas.

Mediante DRX se realiza un análisis composicional a partir de la comparación de los diagramas experimentales obtenidos al analizar la muestra de cerámica arqueológica 2011-4 (Figura 6a) y otra de pigmento 2011-5 (Figura 6b), con diagramas compilados en bases de datos. Se observa que la cerámica arqueológica está constituida principalmente por cuarzo $\left(\mathrm{SiO}_{2}\right)$ y hematita. Las propiedades magnéticas de la hematita fueron comprobadas al acercar la muestra 2011-4 a un imán, lo que verifica el resultado cristalográfico. La muestra 2011-5 está principalmente constituida por cuarzo y goetita.

\section{Discusión}

Los resultados iniciales de la comparación por DRX de la muestra de pigmento amarillo proveniente del sitio Puerto La Tuna (2011-1) con muestras extraídas de potenciales fuentes de aprovisionamiento 

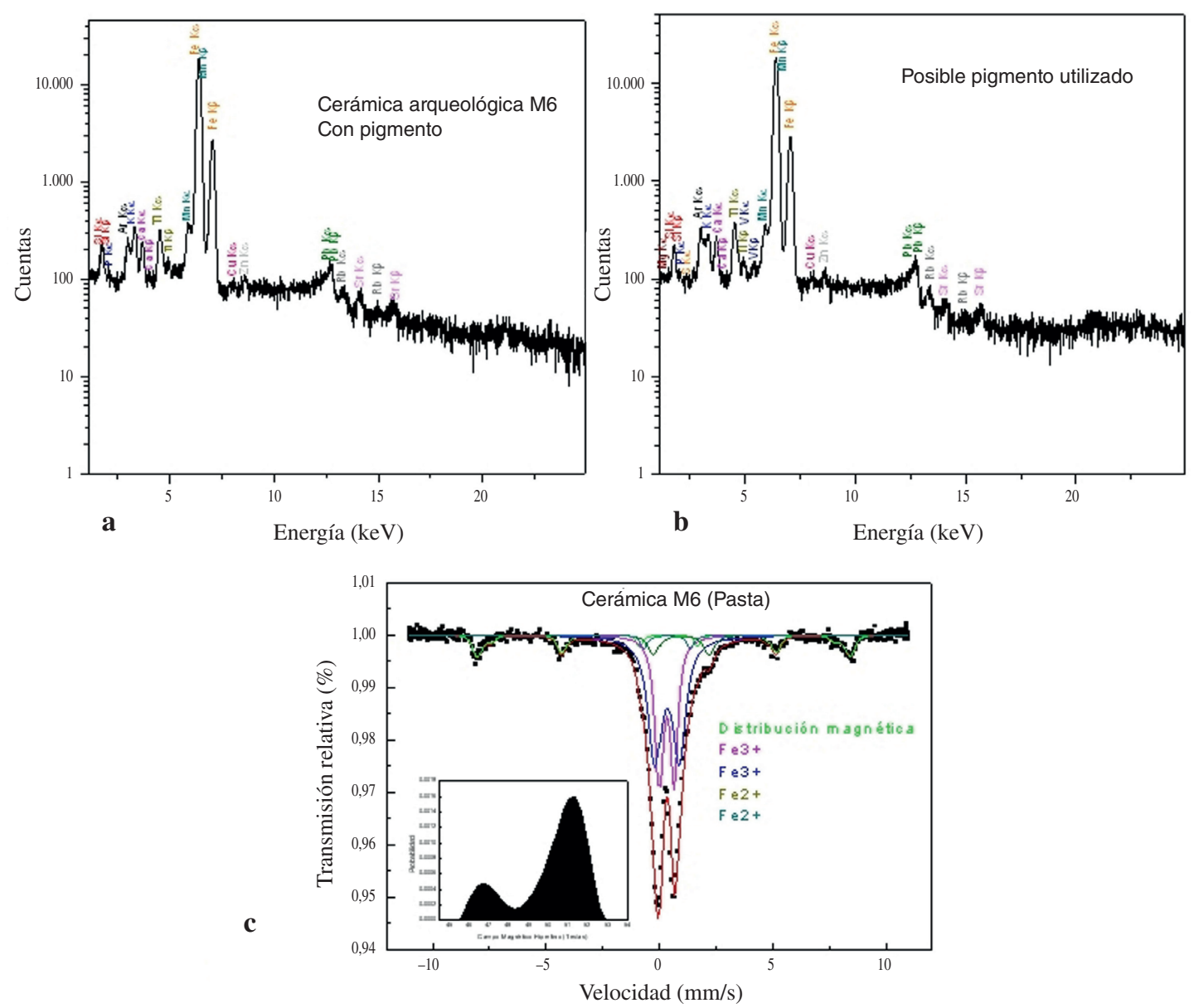

Figura 5. Espectros de FRX; (a) Cerámica (M6) con una superficie pintada, (b) posible pigmento. (c) Espectro Mössbauer a temperatura ambiente de la muestra M6.

XRF spectrum; (a) pottery (M6) with painted surface, (b) possible pigment, (c) Mössbauer spectrum at room temperature of sample M6.

(2011-2 y 3), parecen indicar que algunos pigmentos arqueológicos podrían provenir de la fuente más cercana, ubicada a $20 \mathrm{~km}$ del sitio.

En cuanto al tipo de componentes, los resultados de los análisis de DRX, FRX y EMT efectuados sobre los pigmentos arqueológicos y las muestras de cerámica pintada sugieren que en todos los casos se trata fundamentalmente de hematita y goetita, lo que se acompasa con los elementos identificados a nivel regional (Matarrese et al. 2011, entre otros). Cabe mencionar que cuando la goetita se expone al calor se transforma en hematita cambiando de las tonalidades amarillas a las rojizas (Cornell y Schwertmann 1996). Asimismo la espectroscopia Mössbauer por transmisión realizada sobre una de las muestras (2009-1) da cuenta de la presencia de una distribución de sitios magnéticos que está asociada a diferentes tipos de hematita. Esta diversidad se debe a que, debido a sustituciones isomorfas y a las diferencias en tamaño de partícula, los parámetros hiperfinos de la hematita varían, presentándose una mixtura de sitios que vienen bien representados por una sola distribución magnética. Un doblete de hierro $\mathrm{Fe}_{3}+$ es adjudicado a algún óxido de hierro en estado superparamagnético. El efecto superparamagnético frecuentemente es exhibido por algunos óxidos con tamaño menor de $10 \mathrm{~nm}$ a temperatura ambiente (Cornell y Schwertmann 1996), estos óxidos enmascaran su comportamiento magnético a temperatura ambiente y en lugar de presentar un sexteto en el espectro Mössbauer, presentan un doblete. Los otros dobletes se adjudican a sitios de hierro que forman parte de la estructura de las arcillas. Por último los análisis de FRX en 


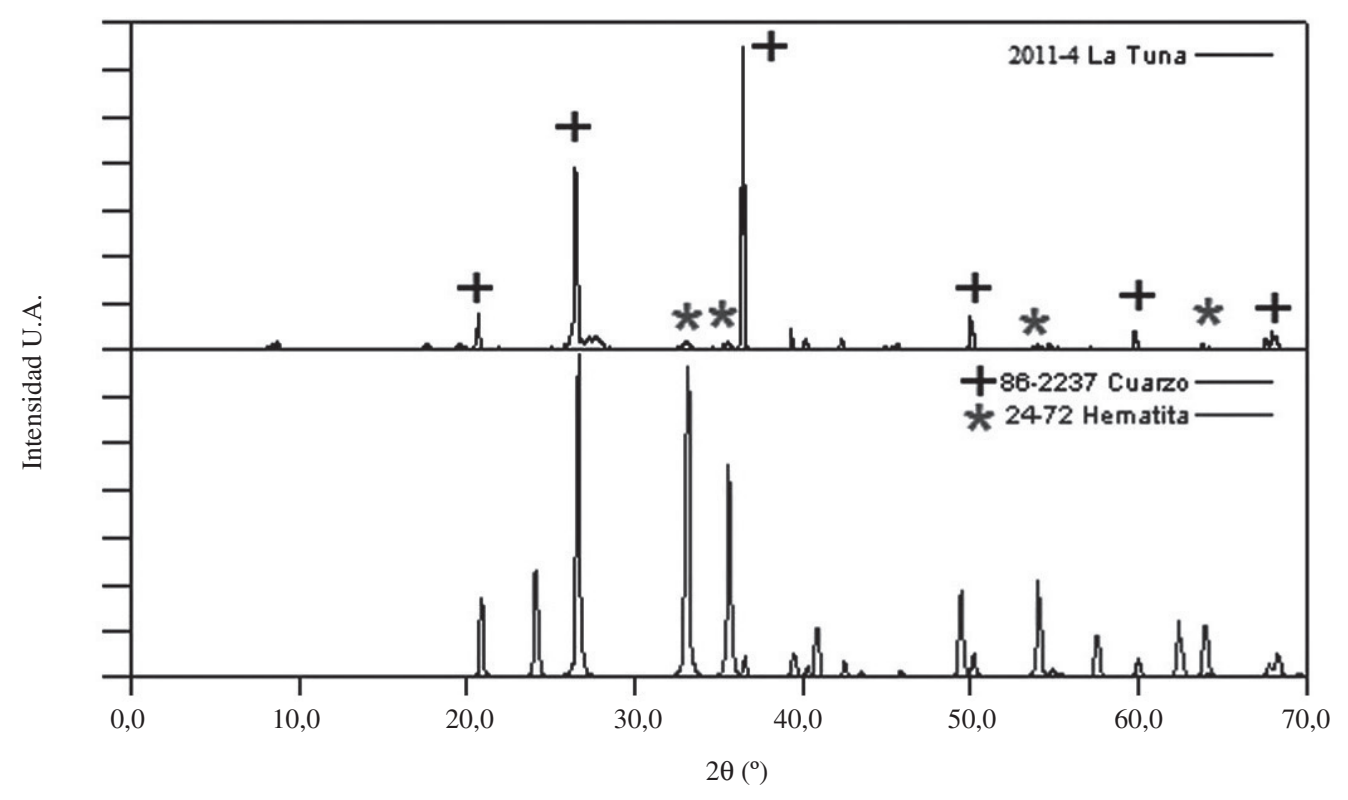

a

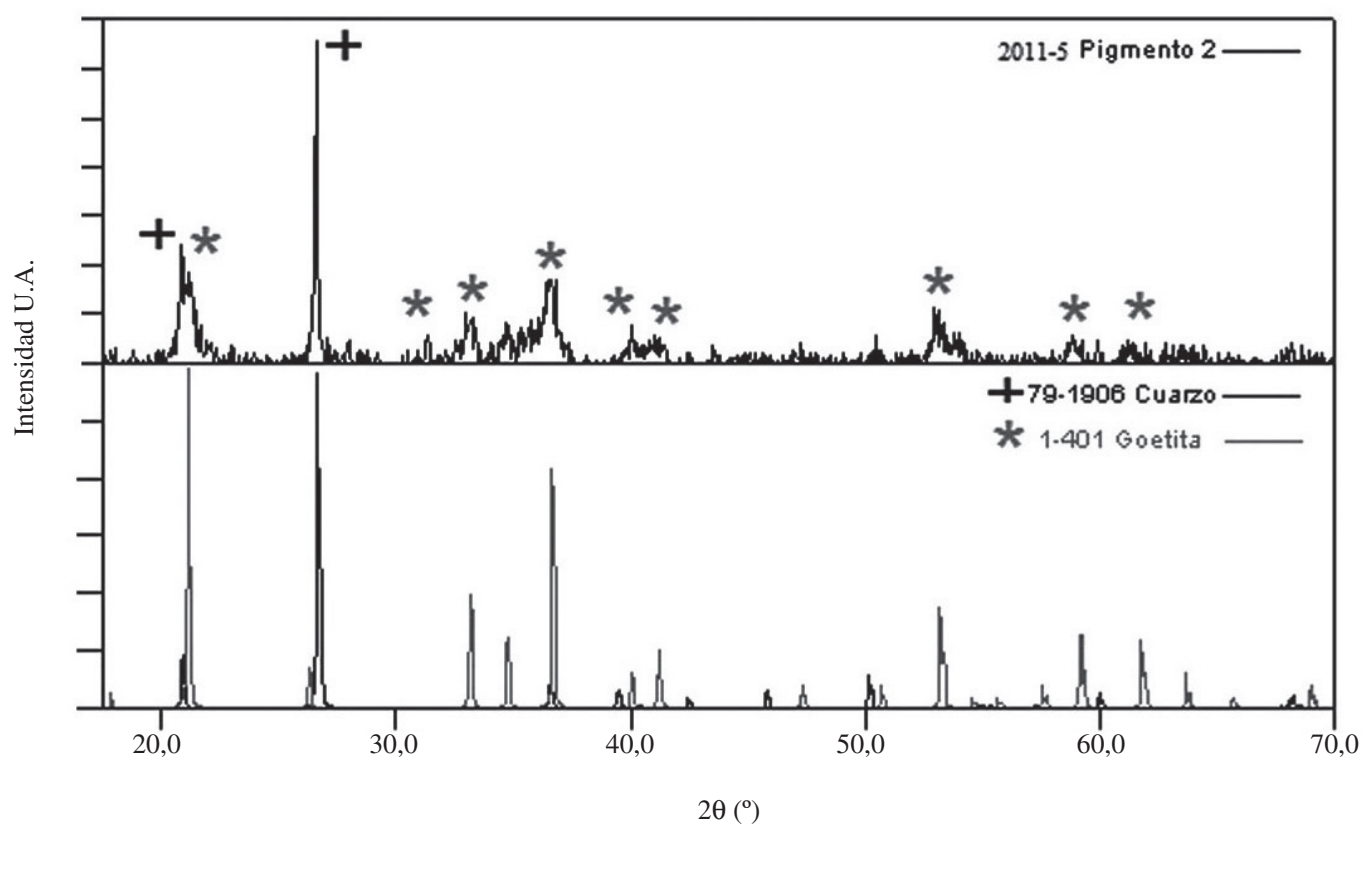

Figura 6. (a) Diagrama de difracción experimental de cerámica arqueológica La Tuna (superior) comparado con los de las muestras minerales de mayor coincidencia compilados en bases de datos (inferiores), (b) Diagrama de difracción experimental de cerámica arqueológica La Tuna (superior) comparado con los de las muestras minerales de mayor coincidencia compilados en bases de datos (inferiores).

(a) Experimental diffraction diagram of La Tuna archaeological ceramic (above) compared with those of the best matching mineral samples compiled into databases (below), (b) experimental diffraction pattern of La Tuna archaeological ceramic (above) compared to best matching mineral samples compiled in databases (below). 
las muestras 2009-1 y 2 descartan la presencia de cinabrio como posible pigmento pues no se identifican azufre ni mercurio.

\section{Conclusiones}

Los resultados de los análisis arqueométricos aquí presentados constituyen una primera aproximación a la caracterización de los pigmentos tanto arqueológicos como potenciales, a su aprovisionamiento y a su uso. La presencia de pigmentos como hematita y goetita en los materiales arqueológicos analizados y en fuentes cercanas al sitio Puerto la Tuna da cuenta de un posible circuito de abastecimiento cercano al sitio. En cuanto a su uso, los resultados de los análisis arqueométricos apoyan los resultados de diferentes líneas de investigación llevadas a cabo en la investigación del área (Beovide 2011a, Malán et al. 2011) que sugieren que el uso de colorantes en el sitio Puerto La Tuna estaría destinado a la decoración de contenedores cerámicos. Sin embargo, por tratarse de un sitio multipropósito no se descartan otros usos de los colorantes vinculados por ejemplo a la funebria. Asimismo, la información proveniente de las excavaciones arqueológicas arroja datos que apuntan a que la fabricación de colorantes podría haberse realizado por lo menos en dos formas: (a) morteros con residuos rojos, en este caso el pigmento fue machacado; (b) desde artefactos pasivos con evidencias de trazas de uso, donde el pigmento fue raspado y picoteado.

Si bien queda mucho camino por andar, este trabajo abre nuevas líneas hacia la caracterización de los pigmentos de origen mineral que permitan profundizar en las redes de circulación de materias primas y en las estrategias de fabricación y uso de colorantes por parte de las sociedades que habitaron la CISL en contextos prehispánicos tardíos.

Agradecimientos: A las instituciones involucradas. Proyecto ANII-FCE2007-186 e investigadores asociados. Especialmente a los evaluadores por sus comentarios enriquecedores.

\section{Referencias Citadas}

Beovide, L. 2011a. La presencia de cultígenos desde el quinto milenio en el registro arqueológico del curso medio platense: revisión y perspectivas. En Avances y Perspectivas en la Arqueología del Nordeste, editado por M. Feuillet, B. Colasurdo, J. Sartori y S. Escudero, pp. 155-175. Servicios Gráficos, Santo Tomé.

Beovide, L. 2011b. Las sociedades prehistóricas de la cuenca del río Santa Lucía: una mirada desde la epistemología de la complejidad. Cuadernos Serie Especial pp. 128-140.

Beovide, L., V. Mata y S. Campos 2007. Los artefactos de molienda en la ocupación humana de la cuenca inferior del río Santa Lucía durante dos eventos transgresivos holocénicos. Actas del XVI Congreso Nacional de Arqueología Argentina, pp. 151-156. Universidad Nacional de Jujuy, San Salvador de Jujuy.

Cornell, R.M. y U. Schwertmann 1996. The Iron oxides, Structure, Properties, Reactions, Ocurrence and Uses. VCH Verlagsgesellschaft mbH, D-69451 Weinheim (Federal Republic of Germany); VCH Publishers, New York.

Di Prado, V., R. Scalise, D. Poiré, J. Canalicchio y L. Gómez 2007. Análisis de elementos colorantes provenientes del sitio Calera (Sierras Bayas, Región Pampeana). Una exploración del uso social y ritual de los pigmentos. En Arqueología en las Pampas, editado por C. Bayón, A. Pupio, M. González, N.
Flegenheimer y M. Frére, pp. 765-780. Sociedad Argentina de Antropología, Buenos Aires.

Lemos, J. y C. Duarte 2011. La Tuna "hace poco": una aproximación al sistema de producción lítica del sitio Puerto La Tuna, Uruguay, hacia ca. 400 años AP. Cuadernos Serie Especial pp. 901-911.

Malán, M., E. Vallvé, A. Malvar y S. Campos 2011. Sobre vasijas y sus decoraciones: un acercamiento a sus funciones y usos. Cuadernos Serie Especial pp. 516-526.

Matarrese, A., V. Di Prado y D. Poiré 2011. Petrologic analysis of mineral pigments from hunter-gatherers archaeological contexts (Southeastern Pampean region, Argentina). Quaternary International 245:2-11.

Peláez, E. 1980. Informe preliminar sobre una pintura rupestre en la Sierra de Mahoma. Actas del III Congreso nacional de Arqueología IV encuentro de Arqueología del Litoral, pp. 87-95. Montevideo.

Penino, R. 1936. Etnografía del Uruguay. Suplemento Dominical de El Día, N173, 10 de mayo, Montevideo.

Porto, J y D. Mazzanti 2007. Pigmentos minerales arqueológicos y fuentes de aprovisionamiento en el sudeste bonaerense: Análisis arqueométrico comparativo. Ponencia presentada en el II Congreso Argentino y I Latinoamericano de Arqueometría. Buenos Aires.

\section{Nota}

1 En todos los casos las muestras se presentan complejas, apreciándose sus porciones cristalinas como constituidas por varias fases. Por este motivo no puede descartarse la presencia de otros componentes en menor proporción a los mayoritarios. 
